\title{
A child chronic cough-specific quality of life measure: development and validation
}

\author{
Peter A Newcombe, ${ }^{1}$ Jeanie K Sheffield, ${ }^{1}$ Helen L Petsky, ${ }^{2}$ Julie M Marchant, ${ }^{2}$ \\ Carol Willis, ${ }^{2}$ Anne B Chang ${ }^{2,3}$
}

- Additional material is published online only. To view please visit the journal online (http://dx.doi.org/10.1136/ thoraxjn-2015-207473).

${ }^{1}$ School of Psychology, University of Queensland, Brisbane, Australia

${ }^{2}$ Qld Children's Respiratory Centre and Qld Children's Medical Research Institute, Royal Children's Hospital, Queensland University of Technology, Brisbane, Australia ${ }^{3}$ Child Health Division, Menzies School of Health Research, Darwin, Australia

\section{Correspondence to} Associate Professor Peter Newcombe, School of Psychology, The University of Queensland, QLD 4072, Australia; newc@psy.uq.edu.au

Research conducted at the Royal Children's Hospital, Brisbane, Australia.

Received 21 June 2015 Revised 16 December 2015 Accepted 8 January 2016 Published Online First 3 February 2016

\section{SLinked}

- http://dx.doi.org/10.1136/ thoraxinl-2015-207315

\section{CrossMark}

$$
\begin{aligned}
& \text { To cite: Newcombe PA, } \\
& \text { Sheffield JK, Petsky HL, } \\
& \text { et al. Thorax 2016;71: } \\
& \text { 695-700. }
\end{aligned}
$$

\section{ABSTRACT \\ Background Quality of life (QoL) measures are an important patient-relevant outcome measure for clinical studies. Cough is the most common symptom that results in new medical consultations. Although adult and parent-proxy cough-specific QoL instruments have been shown to be a useful cough outcome measure, no suitable cough-specific QoL measure for children with chronic cough exists. We report on the statistical properties of a chronic cough-specific QoL (CC-QoL) questionnaire for children.}

Method 130 children (median age 10 years, IQR 8-12 years; 65 girls) participated. A preliminary 37-item version was developed from conversations with children with chronic cough (>4 weeks). Children also completed generic QoL questionnaires (Pediatric QoL Inventory 4.0 (PedsQL4.0), Spence Children's Anxiety Scale (SCAS)) and cough diary scores.

Results The clinical impact method of item reduction resulted in 16 items that had excellent internal consistency (Cronbach's $\alpha=0.94$ ) among these items and also within each domain. Evidence for construct and criterion validity was established with significant correlations between CC-QoL subscales with cough scores, PedsQL and SCAS scores. CC-QoL scores were sensitive to change following an intervention and significant differences were noted between those children coughing and those who had ceased coughing. Minimum important difference (MID) for overall and domain CC-QoL ranged from 0.37-1.36 (distributionbased approach) to 1.11-1.58 (anchor-based approach). Conclusions Chronic cough significantly impacts the QoL of children. The CC-QoL is a reliable, valid and sensitive to change outcome measure that assesses QoL from the child's perspective. Pending data from a confirmatory cohort, a MID for the CC-QoL of 1.1 is recommended when evaluating health status change.

\section{INTRODUCTION}

Patient-related outcomes are considered essential when evaluating health status and clinical outcomes following interventions. The most commonly used patient-related outcome is quality of life (QoL) which has been increasingly integrated into healthcare and employed in a large number of intervention studies in the last three decades. Understanding and measuring QoL is important in realising the burden of disease, in evaluating healthcare interventions, and as an outcome indicator in epidemiological and interventional studies. ${ }^{1}$

\section{Key messages}

What is the key question?

- To determine the psychometric properties and the minimum important difference (MID) of the first child chronic cough-specific quality of life (CC-QoL) measure in children with chronic cough.

\section{What is the bottom line?}

- CC-QoL has convergent and criterion validity, is sensitive to change and its MID has been determined; thus it will be clinically useful for evaluation of interventions.

\section{Why read on?}

- Chronic cough in children is common yet there is no validated child self-reported cough-specific QoL measurement instrument.

The large number of QoL measures available makes choosing an appropriate and relevant instrument difficult. For example, a systematic review identified almost 100 QoL instruments for children and adolescents. ${ }^{1}$ However, QoL is a complex construct and its measurement must include all areas of life and experience relevant to the individual, including the impact of illness and treatment. ${ }^{2}$ Thus, not surprisingly, disease-specific QoL instruments have shown superior specificity and sensitivity over generic QoL instruments ${ }^{3} 4$ and this includes cough-specific QoL instruments for adults $^{5}{ }^{6}$ and parent-proxy cough-specific QoL instruments for children. ${ }^{78}$

In countries where data is available, cough of undifferentiated time length is the most common symptom that results in new medical consultations. ${ }^{9}$ In the USA, 29.5 million office-based doctor visits per year are for cough. ${ }^{9}$ Over recent decades, the impact of cough on patients' QoL has been appreciated resulting in the development of several cough-specific QoL instruments for adults with cough (eg, Leicester Cough Questionnaire ${ }^{5}$ ) and parents of children with cough (eg, parent-proxy cough-specific QoL (PC-QoL) ${ }^{7}{ }^{10}$ ). However, adult QoL instruments are neither suitable for use with children nor appropriate for paediatric use. ${ }^{11}$

Although for adults, there has been an obvious appreciation that patient-related outcomes are patient 'self-reported' outcomes, this same 
Table 1 Impact rank order of the CC-QoL items (items numbered 1-16 included in the final CC-QoL questionnaire)

\begin{tabular}{|c|c|c|c|}
\hline $\begin{array}{l}\text { Item } \\
\text { How often did/were...... }\end{array}$ & Item mean & Proportion endorsing & Impact \\
\hline 1. You hate your cough PSY & 4.72 & 0.72 & 3.38 \\
\hline 2. Coughing annoy you in past week PSY & 4.19 & 0.77 & 3.22 \\
\hline 3. Your cough make you feel frustrated PSY & 3.79 & 0.69 & 2.60 \\
\hline 4. Your cough disturb others $\mathrm{SOC}$ & 3.65 & 0.64 & 2.35 \\
\hline 5. You annoyed by your cough doing activities PSY & 3.54 & 0.60 & 2.11 \\
\hline 6. Your chest or stomach pains bother you PHY & 3.33 & 0.62 & 2.05 \\
\hline 7. Your cough make you feel tired PHY & 3.43 & 0.58 & 1.98 \\
\hline 8. You troubled sleeping at night because of cough PHY & 3.37 & 0.58 & 1.94 \\
\hline 9. Your cough wake you during night PHY & 3.25 & 0.59 & 1.91 \\
\hline 10. You have trouble getting to sleep because of cough PHY & 3.36 & 0.55 & 1.83 \\
\hline 11. Coughing up phlegm annoy you PSY & 3.22 & 0.55 & 1.78 \\
\hline 12. You find it difficult to do things because of cough PHY & 3.18 & 0.55 & 1.73 \\
\hline 13. You feel uncomfortable because of cough PSY & 3.28 & 0.53 & 1.72 \\
\hline 14. Your chest feel bad PHY & 3.13 & 0.54 & 1.68 \\
\hline 15. Your cough annoy others SOC & 3.15 & 0.53 & 1.66 \\
\hline 16. You feel frustrated because you couldn't keep up with others $S O C$ & 3.16 & 0.51 & 1.60 \\
\hline 17. Cough stop you from doing things you like & 2.94 & 0.49 & 1.43 \\
\hline 18. You feel you couldn't keep up with others & 2.79 & 0.47 & 1.31 \\
\hline 19. You stop doing things you like because of cough & 2.84 & 0.46 & 1.30 \\
\hline 20. You annoyed by your chest crackles or rattles & 2.76 & 0.43 & 1.18 \\
\hline 21. Your cough embarrass you & 2.52 & 0.37 & 0.94 \\
\hline 22. Your cough make you feel self-conscious & 2.57 & 0.36 & 0.93 \\
\hline 23. Your cough make you feel worried, troubled & 2.48 & 0.37 & 0.91 \\
\hline 24. You have a headache because of cough & 2.48 & 0.36 & 0.90 \\
\hline 25. Your cough make you feel upset & 2.43 & 0.36 & 0.89 \\
\hline 26. You asked to keep quiet or stop coughing because of cough & 2.27 & 0.33 & 0.74 \\
\hline 27. You feel others stared at you because of your cough & 2.29 & 0.31 & 0.70 \\
\hline 28. Your cough make you feel sad & 2.18 & 0.29 & 0.62 \\
\hline 29. You feel different or left out & 2.07 & 0.27 & 0.57 \\
\hline 30. Your treatment for your cough make you feel different or bad & 2.06 & 0.25 & 0.51 \\
\hline 31. Your family have to turn up the TV volume because of cough & 1.81 & 0.22 & 0.40 \\
\hline 32. You told or asked to go away because of cough & 1.66 & 0.19 & 0.32 \\
\hline 33. You hide while coughing so others didn't see & 1.51 & 0.14 & 0.22 \\
\hline 34. You teased by family and friends because of cough & 1.40 & 0.13 & 0.18 \\
\hline 35. Others shout at you because of cough & 1.42 & 0.10 & 0.14 \\
\hline 36. Someone make fun of you because of cough & 1.30 & 0.06 & 0.08 \\
\hline 37. Someone say they did not want to play with you because of cough & 1.24 & 0.05 & 0.06 \\
\hline
\end{tabular}

recognition has not been afforded to the paediatric population. ${ }^{12}$ While there may be circumstances when a child is too young to complete a QoL instrument (eg, cognitively impaired, ill or fatigued), there is evidence to suggest that children and adolescents aged 5-18 years can reliably and validly self-report their QoL when an age-appropriate measurement instrument is used. ${ }^{13}$

For children younger than 5-6 years, parent-proxy reporting is often the practice of choice. ${ }^{1}$ However, there is ample evidence to suggest that with adult and paediatric populations, information provided by proxy respondents is not equivalent to that reported by the patient. ${ }^{14}{ }^{15}$ Discrepancies between parentproxy and child self-reports have relevance in clinical settings as they can impact on areas to be addressed in therapy. ${ }^{16}$ Indeed the Food and Drug Administration (FDA) notes that "some treatment effects are known only to the patient". ${ }^{17}$ To date, there is no published validated age-related, self-report, cough- specific instrument relevant for children older than 6 years and adolescents who can self-report on their own QoL.

In the absence of an appropriate self-report cough-specific QoL for children and adolescents, we aimed to develop and validate a child chronic cough-specific QoL (CC-QoL) questionnaire in this study. The CC-QoL is designed to assess the impact, on older children and adolescents aged 7-17 years old, of their chronic cough across multiple dimensions of well-being. We also describe the minimum important difference (MID) of the CC-QoL.

\section{METHOD}

Participants

Children aged 7-17 years and newly referred to the Royal Children's Hospital, Brisbane, with chronic cough ( $>4$ weeks) were eligible to participate. All received treatment as per protocols based on standard recommendations and procedures. ${ }^{18}$ 
Children were excluded from the study based on the presence of previously diagnosed respiratory diseases (eg, cystic fibrosis), classical asthma (recurrent wheeze or dyspnoea responsive to $\beta_{2}$-agonist) or other underlying disorders (eg, neurodevelopmental or congenital heart disease).

\section{Materials}

CC-QoL: To obtain relevant items for the CC-QoL, a number of focus group discussions were held with older children and adolescents who had a chronic cough or who had recently sought medical assistance for chronic cough, as was done for our previous PC-QoL. ${ }^{7} 10$ Discussions were audio recorded and verbatim transcripts independently analysed to arrive at a pool of appropriate items. The resultant draft CC-QoL questionnaire included 37 items that assessed the level of frequency of feelings and behaviours related to a child's cough (see table 1). The CC-QoL questionnaire was designed to reflect a number of domains including physical (eg, coughing makes you feel tired), social (eg, others stared at you) and psychological (eg, feel upset). Items referred to a 'past week' recall and were rated on a 7 -point Likert-type scale $(1=$ all the time to $7=$ none of the time) with higher scores reflecting fewer concerns (ie, higher quality of life).

Cough measures: To quantify the severity of the children's cough, a Cough Visual Analogue Scale (Cough VAS) and a Cough Verbal Category Descriptive Scale (VCD) ${ }^{19}$ were included. For both measures, increasing scores reflected greater trouble with, or interference in, their usual activities.

Pediatric QoL Inventory 4.0 (PedsQL): ${ }^{20}$ Participants completed the child version of this 23 -item generic multidimensional questionnaire. The items load on to four QoL dimensions —physical (8 items), emotional (5 items), social (5 items) and school (5 items). Children responded on a 5-point Likert-type scale $(0=$ never a problem to $4=$ almost always a problem $)$. Responses were reverse-scored so that lower scores reflected more negative functioning and were consistent with the scoring direction of the CC-QoL.

Spence Children's Anxiety Scale (SCAS): The SCAS version for older children ${ }^{21} 22$ is a 44-item (including 6 filler items), 4-point scale measure of the child's anxiety relating to six subscales-social phobia, separation anxiety, panic attack/agoraphobia, obsessive-compulsive disorder, generalised anxiety and physical injury fears. The measure has demonstrated good psychometric properties with an internal consistency of 0.92 for the total scale and a 12-week test-retest reliability of $0.63 .^{22}$

\section{Procedure}

At an initial appointment (Time-1), each child completed the CC-QoL questionnaire along with the two measures of cough chronicity-Cough VAS and VCD. ${ }^{19}$ Additionally, the children completed the PedsQL ${ }^{20}$ and SCAS. ${ }^{21}$ All children received a treatment intervention in accordance with the Australian paediatric cough guidelines, ${ }^{18}$ an approach that has recently been tested in a randomised controlled trial. ${ }^{23}$ At a follow-up visit (Time-2), on average 13 weeks post initial visit, children repeated all the questionnaires at the hospital clinic.

\section{Statistical analysis}

We used the clinical impact method as outlined by Juniper ${ }^{24}$ to arrive at a reduced set of items. This involves calculating impact scores that represent the product of an item mean ('Importance') and the proportion of respondents endorsing that item ('Frequency'). Items were then ranked according to these impact scores. Following Juniper ${ }^{24}$ where higher scores reflected greater impact on QoL, our CC-QoL items were reverse scored for these calculations only. That is, higher numbers indicated greater difficulties with well-being. The criteria for inclusion of an item in the final CC-QoL questionnaire was endorsement of the impact of that item by $\geq 50 \%$ of participants along with a natural break in rank-ordered impact scores.

Non-parametric analyses are reported as the data were not normally distributed. Descriptive data are presented as medians and IQR; Mann-Whitney $U$ test was used for unpaired comparisons and Wilcoxon test for paired comparisons; Spearman's correlation $\left(r_{s}\right)$ was used to determine relationships between variables. Cronbach's $\alpha$ was used to assess the internal consistency of the items. Scores on $\alpha$ can vary between 0 and 1 with high values (generally $>0.8$ ) indicating that the items contribute to an underlying construct. Test-retest reliability was assessed with intraclass correlation coefficient (ICC). Data were analysed using SPSS software V.21.

Two methods were adopted for MID calculations (see online supplement file): (A) a distribution method based on criteria that link important differences to a statistical parameter and include effect size (ES), ${ }^{25} \mathrm{SEM}^{26}$ and a half $\mathrm{SD},{ }^{27}$ as minimally important difference estimates; and (B) an anchor-based approach $^{28}$ where changes in QoL were examined against changes in an external but meaningful anchor, cough severity, using VCD. A positive QoL change score (ie, higher scores at Time-2 than at Time-1) represented an improved QoL. The anchor change scores were derived from the differences in ratings of VCD at the two time points with classification schema based on previous research ${ }^{28} 29$ and seen as a change of visible difference. We previously classified using anchor values. ${ }^{30}$

\section{RESULTS}

One hundred and thirty children (median age 10 years, IQR 812 years; 65 girls) participated in this study. Protracted bacteria bronchitis was diagnosed in 42 children (32.3\%), asthma in 19 $(14.6 \%)$ and tracheomalacia in 15 (11.5\%). Preliminary analyses were conducted to ensure that the children with protracted bacteria bronchitis did not differ from other participants. No significant differences in age or in the PedsQL scores at Time-1 or Time- 2 were evident so the data across the differing respiratory conditions could thus be reliably combined.

\section{Item reduction}

Table 1 presents the rank ordering of the 37 QoL items according to the strength of their impact. With an inclusion criteria set at $\geq 50 \%$ endorsement of an item together with a natural break in impact rating, the 16 highest impact scores were selected. Based on table 1 data, this reduced CC-QoL scale was internally consistent, $\alpha=0.94$, with a median score of 4.63 (IQR 3.55.56). Interitem correlations ranged from $r_{s}=0.11$ to $r_{s}=0.83$ $($ mean $=0.51)$. This internal consistency was replicated at 13 weeks with $\alpha=0.97$, median score of 6.75 (IQR 5.81-7) and interitem correlations ranging from $r_{s}=0.23$ to $r_{s}=0.87$ (mean $=0.65)$. Further, the 13 -week test-retest reliability was moderate, $\mathrm{ICC}=0.40$. Age was not significantly related to CC-QoL total scores either at Time- $1, \mathrm{r}_{\mathrm{s}}=-0.10, \mathrm{p}=0.347$ or at Time-2, $\mathrm{r}_{\mathrm{s}}=0.21, \mathrm{p}=0.076$.

\section{Domains}

Based on the WHO definition of health, the 16 items of the CC-QoL questionnaire were allocated into Physical (physical manifestation or physical consequence of cough), Social (outcome or consequence in the social domain or a response of others to the cough) and Psychological (feeling, reaction or 
emotional response related to the cough) domains, as previously done. ${ }^{10}$ Seven items were allocated to the Physical domain; three to the Social domain; and six to the Psychological domain (see table 1). Face validity of each item to their allocated domain was determined by the expert opinion of a panel of respiratory clinicians and psychologists.

All three domains were shown to be internally consistent with $\alpha$ ranging from 0.76 to 0.90 (Time-1) and 0.87 to 0.95 (Time-2). Item-total correlations (lowest: 0.42-0.72 for Social domain at Time-1) and test-retest reliability (ICCs: 0.39-0.45) further support the reliability of the domains.

\section{Validity}

Construct (convergent) and criterion (concurrent) validity for the CC-QoL full scale and domain scores were examined through correlations with VCD, VAS and the subscales of the PedsQL and the SCAS (table 2). For the full scale CC-QoL, convergent validity was demonstrated with significant correlations with the VCD and VAS at Time-1, $\mathrm{r}_{\mathrm{s}} \geq-0.44, \mathrm{p} \leq 0.02$, and Time-2, $\mathrm{r}_{\mathrm{s}} \geq-0.89, \mathrm{p} \leq 0.001$. Higher cough scores were related to poorer quality of life. Concurrent validity at Time- 1 was evidenced with significant correlations with all the PedsQL subscale scores, $r_{s} \geq 0.32, p \leq 0.003$, such that higher ratings of QoL across each of the PedsQL domains was related to child reporting of higher cough-related QoL. For the SCAS, only the correlations between child-reported panic and social phobia with QoL reached significance, $r_{s} \geq 0.33, p \leq 0.043$. In all cases, higher anxiety scores were related to poorer quality of life. As can be seen in table 2 , and although generally weaker, similar significant correlations at Time- 2 with the PedsQL further confirm the psychometric qualities of the CC-QoL.

For the domain scores of the CC-QoL, there were significant correlations with VAS and VCD across all domains and time points (except for the Social domain with VAS at Time-1, $\mathrm{r}_{\mathrm{s}}=$ $-0.34, \mathrm{p}=0.078$ ). The correlations with the PedsQL subscale scores further confirm the concurrent validity of the CC-QoL domains (see table 2).

\section{Sensitivity to change}

The CC-QoL measure was sensitive to change across time following intervention. Descriptive data (medians with IQRs) along with Wilcoxon tests of difference are presented in table 3. All domain and full scale scores showed significant improvement in child-reported QoL following the intervention, all $\mathrm{p}<0.001$. Further evidence for this sensitivity is demonstrated when the CC-QoL domain and full scale scores for those who had and had not ceased coughing at Time- 2 were compared. Descriptive data (medians with IQRs) and the Mann-Whitney tests of difference are presented in table 4 and show that those children who had not ceased coughing reported significantly lower QoL than did those children who had ceased coughing, all $\mathrm{p}<0.001$.

\section{Minimal important difference}

For the distribution-based approach of computing MID, methods were based on computing the ES, the SEM and the SD of the sample in relation to the final 16-item CC-QoL scores as well as the domains. ES MID for the 16-item scale was 1.27 and ranged from 1.10 to 1.36 for the domain QoLs. With $\alpha=0.94$ and baseline $\mathrm{SD}=1.50$, the SEM for the 16-item CC-QoL instrument was 0.37. SEMs for the QoL domains ranged from 0.51 to 0.86 . Half the SD for overall QoL was 0.75 with the domains ranging from 0.78 to 0.81 .

Based on the anchor-based calculation method (ie, equivalent to a 'small' change in VCD), an MID of 1.44 was suggested for the 16-item CC-QoL instrument and ranged from 1.11 to 1.58 for the domains. Given that MID is the 'minimal' difference that would indicate a change of importance, a value of 1.1 was therefore selected. Based on this value, 11 of the 12 children who had a valid VCD change score $\geq 1$ would have been identified with a clinically important QoL change score.

\section{DISCUSSION}

This study sought to generate a reliable and valid paediatric QoL instrument for children with chronic cough. The CC-QoL instrument is an important addition to the suite of QoL instruments as it measures the impact of cough on a child's own reported well-being and burden across multiple dimensions. The original 37 items were developed through focus groups and interviews with children and adolescents and thus reflected issues of relevance to those directly impacted by the cough. The clinical impact method identified 16 items that were reported by the children to make the greatest impact on their lives. Overall, the final 16-item CC-QoL instrument was psychometrically sound as were the domains. All had excellent internal consistency and moderate test-retest reliability, correlated with a generic QoL instrument and cough measures (thus valid), and were sensitive to change (significant difference demonstrated following resolution of the child's cough post intervention). Although there was a wide age range with the children in this study, age was not significantly related to CC-QoL scores.

We determined the MID for the CC-QoL instrument using two approaches to assist clinicians and researchers in interpreting meaningful changes in health-related QoL in children with chronic cough. Using the distribution-based method, the MID for overall QoL ranged from 0.37 to 1.27, while the anchorbased method established the MID as 1.44 for overall QoL and 1.11-1.58 for the domains. The larger MIDs derived from anchor-based methods exceeded the distribution-based MIDs and are supported by our previous research with $\mathrm{PC}-\mathrm{QoL}^{30}$ and by Kolotkin et al's ${ }^{31}$ research with severe initial impairments in obesity-specific QoL. Nonetheless, that the values from the two methods differed and were higher than our previous PC-QoL values calls into question as to which MID value would be most appropriate for clinical and research use. As reported elsewhere, each method has advantages and disadvantages. ${ }^{28} 32$ The distribution-based approach uses the sample statistics, which are relatively easy to generate, to establish an MID. The anchorbased method emphasises the patient's individual opinion where the 'anchor' represents change across an independent measure. Often a single global change rating scale is used as the anchor ${ }^{28}$ however this method has been criticised as being highly correlated to the patient's present state. ${ }^{33}$ We therefore employed change across Time-1-2 VCD scores as the anchor, a method previously used by us ${ }^{30}$ and others. ${ }^{34}$ Based on this approach that is patient-driven, an MID of 1.1 would seem appropriate as this value represents the minimal change across the domains and overall QoL. This value would have identified 92\% (11/12) of our selected sample with complete data at Time- 2 as achieving a clinically important difference in their QoL. It should be noted that our MID is based on a small sample size and is samplespecific. Therefore, more research with larger and separate cohorts of children with chronic cough is needed to validate this figure.

Adult cough-specific QoL questionnaires have been used for several years. ${ }^{5}$ However, the use of adult measures with paediatric populations is generally inappropriate. ${ }^{11}$ For the area of cough for example, the inclusion of items such as 'My cough has interfered with my job' is not relevant to children. As with 
Table 2 Spearman's correlations of domain and total CC-QoL scores with cough measures and SCAS and PedsQL subscale scores at initial (T1) and 13-week follow-up (T2) visits

\begin{tabular}{|c|c|c|c|c|c|c|c|c|}
\hline & \multicolumn{8}{|l|}{ CC-QoL } \\
\hline & \multicolumn{2}{|l|}{ Physical } & \multicolumn{2}{|l|}{ Social } & \multicolumn{2}{|c|}{ Psychological } & \multicolumn{2}{|l|}{ Total } \\
\hline & $\mathrm{T1}$ & $\mathrm{T} 2$ & $\mathrm{~T} 1$ & $\mathrm{~T} 2$ & $\mathrm{T1}$ & $\mathrm{T} 2$ & $\mathrm{~T} 1$ & $\mathrm{~T} 2$ \\
\hline \multicolumn{9}{|l|}{ Cough measures } \\
\hline Cough VCD & $-0.53^{* *}$ & $-0.87^{* * *}$ & $-0.40^{*}$ & $-0.68^{* *}$ & $-0.49 * *$ & $-0.88^{* * *}$ & $-0.48^{*}$ & $-0.89 * * *$ \\
\hline Cough VAS & $-0.45^{* *}$ & $-0.91 * * *$ & $-0.34^{\#}$ & $-0.80^{* *}$ & $-0.43^{*}$ & $-0.93^{* * *}$ & $-0.44^{*}$ & $-0.89 * * *$ \\
\hline \multicolumn{9}{|l|}{ SCAS } \\
\hline Social phobia & $-0.47^{* *}$ & -0.30 & $-0.32^{\#}$ & -0.22 & -0.27 & -0.24 & $-0.37^{*}$ & -0.19 \\
\hline Separation anxiety & -0.04 & -0.07 & -0.22 & $-0.32^{\#}$ & -0.07 & -0.13 & -0.02 & -0.05 \\
\hline Panic attack/agoraphobia & $-0.36^{*}$ & -0.13 & -0.23 & -0.26 & $-0.32^{\#}$ & -0.13 & $-0.33^{*}$ & -0.12 \\
\hline Obsessive-compulsive disorder & -0.24 & -0.14 & -0.07 & -0.22 & -0.26 & -0.06 & -0.25 & -0.12 \\
\hline Generalised anxiety & -0.26 & -0.19 & $-0.32^{\#}$ & -0.30 & -0.19 & -0.15 & -0.24 & -0.16 \\
\hline Physical injury fears & 0.04 & -0.15 & 0.03 & -0.33 & 0.13 & -0.25 & -0.11 & -0.26 \\
\hline \multicolumn{9}{|l|}{ PedsQL4.0 } \\
\hline Psychosocial & $0.49 * * *$ & 0.32 ** & $0.34^{* *}$ & $0.33^{* *}$ & $0.45^{* * *}$ & $0.24^{\#}$ & $0.48^{* * *}$ & $0.30^{*}$ \\
\hline Physical & $0.52^{* * *}$ & $0.66^{* * *}$ & $0.24^{*}$ & $0.60 * * *$ & $0.36^{* *}$ & $0.58 * * *$ & $0.44^{* * *}$ & 0.62 *** \\
\hline Emotional & $0.44^{* * *}$ & $0.29 *$ & 0.20 & $0.32 * *$ & $0.39 * * *$ & $0.20^{\#}$ & $0.41 * * *$ & $0.26^{*}$ \\
\hline Social & $0.32^{* *}$ & $0.26^{*}$ & $.022^{*}$ & 0.13 & $0.29 * *$ & 0.09 & $0.32 * *$ & 0.16 \\
\hline School & $0.37^{* *}$ & $0.24^{\#}$ & $0.38^{* * *}$ & $0.26^{*}$ & $0.36^{* *}$ & $0.25^{*}$ & 0.40 *** & $0.26^{*}$ \\
\hline
\end{tabular}

all measures, QoL instruments should accurately reflect the concept they purport to measure. Non-valid measures may wrongly represent the impact of treatments and interventions on a range of dimensions for the individual patient or their broader network.

Our own PC-QoL instrument ${ }^{7} 81030$ is a psychometrically sound parent-proxy measure for use in families with a very young child with chronic cough and has been translated into four languages. However, the evidence suggests that proxy-reporting and child self-reporting of QoL are not equivalent. ${ }^{14}$ Further, Varni et $a l^{13}$ note that child patient-reported outcomes (ie, 'the voices of children') should be the standard when measuring matters pertaining to their health and wellbeing. Hence, we sought to develop a child-specific chronic cough QoL as this was a clinical research gap. This was also articulated in a recent review of the available tools for assessing

Table 3 Preintervention and postintervention median scores (with IQR in brackets) for CC-QoL full scale and domain scores and for VCD and VAS with Wilcoxon signed-rank tests of difference $(\mathrm{N}=68)$

\begin{tabular}{|c|c|c|c|}
\hline Domains & Preintervention & Postintervention & Wilcoxon test (Z) \\
\hline VCD & $3.00(2.00-4.00)$ & $0.00(0.00-2.00)$ & $2.47^{*}$ \\
\hline VAS & $5.00(4.00-7.00)$ & $1.00(1.00-3.75)$ & $2.63^{* *}$ \\
\hline \multicolumn{4}{|l|}{ CC-QoL } \\
\hline Physical & $4.86(3.43-6.14)$ & $6.71(6.00-7.00)$ & $5.83^{* * *}$ \\
\hline Social & $4.67(3.33-6.33)$ & $7.00(6.00-7.00)$ & $5.25^{* * *}$ \\
\hline Psychological & $4.00(2.83-5.67)$ & $6.83(5.50-7.00)$ & $6.32^{* * *}$ \\
\hline Total & $4.63(3.5-5.56)$ & $6.75(5.81-7.00)$ & $6.25^{* * *}$ \\
\hline
\end{tabular}

outcomes in studies of chronic cough. ${ }^{35}$ The reliable and validated CC-QoL reported in this study, with further testing, can become a valuable tool in the evaluation of future treatment and clinical research and therefore contribute to improving QoL of children with chronic cough.

This study is not without its limitations. The self-report measures (eg, VCD, VAS) used as an outcome against which validation was determined and as an anchor for MID can suffer from response biases and therefore their use is questionable. Indeed, the correlations between CC-QoL scores and the VCD and VAS were significantly higher at Time- 2 than at Time- 1 which might provide evidence for biased reporting. However, self-reported cough measures have been shown to be valid indicators of objective cough metered counts and can reliably reflect changes in cough. ${ }^{19}$ The age range of our cohort was large with likely different comprehension skills. However, the youngest

Table 4 Median scores (with IQR in brackets) for CC-QoL full scale and domain scores for those children and adolescents who did $(n=48)$ and did not $(n=15)$ cease coughing post intervention together with Mann-Whitney tests of difference

\begin{tabular}{|c|c|c|c|}
\hline \multirow[b]{2}{*}{ Domains } & \multicolumn{2}{|c|}{$\begin{array}{l}\text { Ceased coughing post } \\
\text { intervention }\end{array}$} & \multirow[b]{2}{*}{ Mann-Whitney (Z) } \\
\hline & No & Yes & \\
\hline \multicolumn{4}{|l|}{ CC-QoL } \\
\hline Physical & $6.00(4.86-6.43)$ & $7.00(6.52-7.00)$ & $4.05^{* *}$ \\
\hline Social & $5.33(3.67-7.00)$ & $7.00(6.67-7.00)$ & $3.78^{* *}$ \\
\hline Psychological & $5.17(3.33-6.00)$ & $7.00(6.54-7.00)$ & $5.06^{* *}$ \\
\hline Total & $5.44(4.44-6.25)$ & $7.00(6.52-7.00)$ & $4.81^{* *}$ \\
\hline
\end{tabular}


children (7 years) are consistent with previous research ${ }^{36}$ that has found this age group reliable and accurate in understanding and responding to similarly worded items. Our sample of children was mainly young $(75 \% \leq 11$ years) and this may have masked any age-related changes in QoL. It remains for future research to explore this further. The variation in the MIDs calculated under the different approaches raises the question of which is the most appropriate for use in clinical and research practices. Continuing and more varied sample research in this area is necessary.

As with all QoL tools, the CC-QoL questionnaire is a subjective measure and, as such, should not be expected to replace objective measures of cough. But, it will reveal the severity of the impact of cough from the perspective of the person who matters-the child patient-and therefore should be seen as a necessary complement for a patient-related outcome in illnesses where cough is the dominant symptom. The importance of measuring the impact of cough symptoms on well-being can provide key insights into the overall management of cough in paediatrics. Although, the CC-QoL questionnaire was shown to be reliable, valid and sensitive to change, more work needs to be completed to validate the discriminant utility of the measure.

Acknowledgements The authors thank the parents and children who participated in this study. The authors also thank Drs Brent Masters, Paul Francis, Alan Isles and Helen Buntain for allowing them to enrol their patients in this study.

Funding The study was supported by a programme grant from the Queensland Children's Medical Research Institute (Australia) and ABC is supported by an NHMRC (Australia) Practitioner fellowship (1058213).

Competing interests None declared.

Patient consent Parental consent was obtained.

Ethics approval Royal Children's Hospital Human Ethics Committee, Brisbane, Australia.

Provenance and peer review Not commissioned; externally peer reviewed.

\section{REFERENCES}

1 Solans M, Pane S, Estrada MD, et al. Health-related quality of life measurement in children and adolescents: a systematic review of generic and disease-specific instruments. Value Health 2008;11:742-64.

2 Calman KC. Quality of life in cancer patients_an hypothesis. J Med Ethics 1984;10:124-7.

3 Wiebe $S$, Guyatt $G$, Weaver $B$, et al. Comparative responsiveness of generic and specific quality-of-life instruments. J Clin Epidemiol 2003;56:52-60.

4 Kalpaklioglu AF, Kara T, Kurtipek E, et al. Evaluation and impact of chronic cough: comparison of specific vs generic quality-of-life questionnaires. Ann Allergy Asthma Immunol 2005;94:581-5.

5 Birring SS, Prudon B, Carr AJ, et al. Development of a symptom specific health status measure for patients with chronic cough: Leicester Cough Questionnaire (LCQ). Thorax 2003;58:339-43.

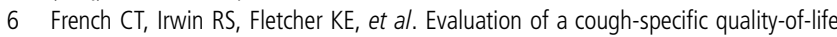
questionnaire. Chest 2002:121:1123-31.

7 Newcombe PA, Sheffield JK, Juniper EF, et al. Development of a parent-proxy quality-of-life chronic cough-specific questionnaire: clinical impact vs psychometric evaluations. Chest 2008;133:386-95.

8 Newcombe PA, Sheffield JK, Chang AB. Parent cough-specific quality of life: development and validation of a short form. J Allergy Clin Immunol 2013; 131:1069-74

9 Irwin RS. Introduction to the diagnosis and management of cough: ACCP evidence-based clinical practice guidelines. Chest 2006;129(Supp):25S-27S.

10 Newcombe PA, Sheffield JK, Juniper EF, et al. Validation of a parent-proxy quality of life questionnaire for paediatric chronic cough (PC-QOL). Thorax 2010;65:819-23.

11 Landgraf JM, Abetz I. Measuring health outcomes in pediatric populations: issues in psychometrics and application. In: Spilker B, ed. Quality of life and pharmacoeconomics in clinical trials. Philadelphia: Lippincott-Raven, 2004:793-802.

12 Clarke SA, Eiser C. The measurement of health-related quality of life (QOL) in paediatric clinical trials: a systematic review. Health Qual Life Outcomes 2004:2:66.

13 Varni JW, Limbers C, Burwinkle TM. Literature review: health-related quality of life measurement in pediatric oncology: hearing the voices of the children. J Pediatr Psychol 2007;32:1151-63.

14 Chang PC, Yeh CH. Agreement between child self-report and parent proxy-report to evaluate quality of life in children with cancer. Psychooncology 2005; 14:125-34

15 Sprangers MAG, Aaronson NK. The role of health care providers and significant others in evaluating the quality of life of patients with chronic disease: a review. J Clin Epidemiol 1992:45:743-60.

16 Johnston CA, Steele RG, Herrera EA, et al. Parent and child reporting of negative life events: discrepancy and agreement across pediatric sample. J Pediatr Psychol 2003;28:579-88.

17 FDA. Guidance for industry: patient-reported outcome measures: use in medical product development to support labeling claims. Rockville, MD: Center for Drug Evaluation and Research, Food and Drug Administration, 2006.

18 Chang $A B$, Landau LI, Van Asperen PP, et al. Cough in children: definitions and clinical evaluation. Position statement of the Thoracic Society of Australia and New Zealand. Med J Aust 2006;184:398-403.

19 Chang $A B$, Newman RG, Carlin J, et al. Subjective scoring of cough in children: parent-completed vs child-completed diary cards vs an objective method. Eur Respir J 1998;11:462-6.

20 Varni JW, Burwinkle TM, Seid M, et al. The PedsQL 4.0 as a pediatric population health measure: feasibility, reliability, and validity. Ambul Pediatr 2003;3:329-41.

21 Spence SH. A measure of anxiety symptoms among children. Behav Res Ther 1998;36:545-66

22 Spence SH, Barrett PM, Turner CM. Psychometric properties of the Spence Children's Anxiety Scale with young adolescents. Anxiety Disord 2003;17:605-25.

23 Chang $A B$, Robertson $C F$, van Asperen PP, et al. A cough algorithm for chronic cough in children: a multicenter, randomized controlled study. Pediatrics 2013;131: e1576-83

24 Juniper EJ, Guyatt GH, Streiner DL, et al. Clinical impact versus factor analysis for quality of life questionnaire construction. J Clin Epidemiol 1997;50:233-8.

25 Wyrwich KW, Wolinsky FD. Identifying meaningful intra-individual change standards for health-related quality of life measures. J Eval Clin Pract 2000;6:39-49.

26 Wyrwich KW. Minimal important difference thresholds and the standard error of measurement: Is there a connection? J Biopharm Stat 2004;14:97-110.

27 Norman GR, Sloan JA, Wyrwich KW. Interpretation of changes in health-related quality of life: the remarkable universality of half a standard deviation. Med Care 2003:41:582-92.

28 Juniper EF, Guyatt GH, Willan A, et al. Determining a minimally important change in a disease-specific Quality of Life Questionnaire. J Clin Epidemiol 1994:47:81-7.

29 Raj AA, Pavord DI, Birring SS. Clinical cough IV: what is the minimal important difference for the Leicester Cough Questionnaire? Handb Exp Pharmacol 2009;187:311-20.

30 Newcombe PA, Sheffield JK, Chang AB. Minimally important change in a parent-proxy quality of life questionnaire for pediatric chronic cough. Chest 2011;139:576-80.

31 Kolotkin RL, Crosby RD, Williams GR. Integrating anchor-based and distribution-based methods to determine clinically meaningful change in obesity-specific quality of life. Qual Life Res 2002;11:670.

32 Brozek JL, Guyatt GH, Schünemann HJ. How a well-grounded minimal important difference can enhance transparency of labelling claims and improve interpretation of a patient reported outcome measure. Health Qual Life Outcomes 2006:4:69-75.

33 Norman G, Stratford P, Regehr G. Methodological problems in the retrospective computation of responsiveness to change: the lessons of Cronbach. J Clin Epidemiol 1997; 50:869-79.

34 Eton DT, Cella D, Yost KJ, et al. A combination of distribution- and anchor-based approaches determined minimally important differences (MIDs) for four endpoints in a breast cancer scale. J Clin Epidemiol 2004;57:898-910.

35 Boulet LP, Coeytaux RR, McCrory DC, et al. Tools for assessing outcomes in studies of chronic cough: CHEST guideline and expert panel report. Chest 2015:147:804-14.

36 Juniper EF, Guyatt $\mathrm{GH}$, Feeny $\mathrm{DH}$, et al. Minimum skills required by children to complete health-related quality of life instruments for asthma: comparison of measurement properties. Eur Respir J 1997;10:2285-94. 